\title{
CONOCIMIENTOS, ACTITUDES Y PRÁCTICAS DE ESCOLARES PARA PREVENIR EL DENGUE: UNA REVISIÓN NARRATIVA
}

Estéfani Alejandra Buitrago Rodríguez ${ }^{1}$, Anderson Rocha Buelvas ${ }^{2}$

\section{Resumen}

Introducción. Para evaluar la efectividad de los programas de prevención aplictitudes y prácticas (CAP). Por ello, surgió la necesidad de evidenciar la literatura existente sobre los conocimientos, actitudes y prácticas del dengue en escolares.

Métodos. Se presenta una revisión de literatura utilizando como fuentes Pubmed, ScienceDirect y Scielo. Se incluyen 16 artículos publicados entre 2001-2014.

Resultados. La mayoría de los estudios revelaron que los conocimientos, actitudes y estrategias de prevención de los escolares frente al dengue son insuficientes, contradictoriamente, la implementación de estrategias educativas promovían un cambio positivo en ellos.

Conclusión. Los estudios CAP han demostrado que las estrategias de prevención del dengue funcionan, sin embargo el impacto positivo solo es tangible en la medida que estas acciones tienen continuidad.

Palabras clave: dengue, salud escolar, conocimientos, actitudes y práctica en salud, países en desarrollo, efficacy (DeCS)

\footnotetext{
${ }^{1}$ Bacterióloga y laboratorista clínico. Universidad Colegio Mayor de Cundinamarca. Magister en salud pública y desarrollo social. Fundación Universitaria del Área Andina. Correo electrónico de contacto: nenaleja61@hotmail.com

${ }^{2}$ Odontólogo. Docente, Maestría en salud pública y desarrollo social, Fundación Universitaria del Área Andina-Sede Bogotá.
} 


\section{KNOWLEDGE, ATTITUDES AND PRACTICES OF SCHOOLCHILDREN TO PREVENT DENGUE FEVER: A NARRATIVE REVIEW}

Estéfani Alejandra Buitrago Rodríguez, Anderson Rocha Buelvas

\section{Abstract}

Introduction. To evaluate the effectiveness of prevention programs applied in some developing regions of diseases such as dengue, tools such as surveys on knowledge, attitudes and practices (KAP) are used. Therefore, it became necessary to highlight the existing literature on knowledge, attitudes and practices of dengue in schoolchildren.

Methods. A review of the literature using the sources Pubmed, ScienceDirect and Scielo is submitted. It includes 16 articles published between 2001 and 2014.

Results. Most studies revealed that the schoolchildren knowledge, attitudes and prevention strategies against dengue are insufficient, contradictorily, the implementation of educational strategies promote a positive change in them.

Conclusion. CAP Studies have shown that dengue prevention strategies are successful, however the positive impact work is tangible only to the extent that these actions have continuity. 


\section{CONHECIMENTO, ATITUDES E PRÁTICAS DOS ESTUDANTES ESCOLARES PARA PREVENIR A DENGUE: UMA REVISÃO NARRATIVA}

Estéfani Alejandra Buitrago Rodríguez, Anderson Rocha Buelvas

\section{Resumo}

Introdução. Para avaliar a eficácia dos programas de prevenção aplicados em algumas regiões em desenvolvimento para doenças como a dengue, ferramentas, tais como pesquisas sobre conhecimentos, atitudes e práticas (CAP) são utilizadas. Portanto, tornou-se necessário destacar a literatura existente sobre conhecimentos, atitudes e práticas sobre a dengue nos estudantes escolares.

Metodologia. Uma revisão da literatura utilizando fontes como Pubmed, ScienceDirect e Scielo é apresentada. 16 artigos publicados entre 2001-2014 estão incluídos.

Resultados. A maioria dos estudos revelaram que os conhecimentos, atitudes e estratégias de prevenção dos estudantes escolares contra a dengue são insuficientes; contraditoriamente, a implementação de estratégias educacionais promoveu mudanças positivas neles.

Conclusão. Estudos CAP têm demonstrado que as estratégias de prevenção da dengue funcionam, no entanto, o impacto positivo é tangível apenas na medida em que essas ações têm continuidade. 


\section{Introducción}

El dengue es una enfermedad considerada de gran impacto a nivel epidemiológico, social y económico, razón por la que constituye un problema creciente y un reto para la salud pública mundial. Se estima que 2,5 billones de personas se encuentran en zonas endémicas (1). El reporte más antiguo de una posible epidemia data de los años 1779 y 1780 , cuando una epidemia azotó Asia, África y América del norte (2). Los brotes de dengue generan mucha preocupación para países en desarrollo como Colombia y otros países del trópico, no solo por ser países endémicos sino porque no siempre la implementación de estrategias preventivas contribuye a disminuir la aparición de nuevos casos y la tasa de mortalidad infantil(3).

Según la OMS, esta enfermedad ha tenido índices de propagación elevados en todas las regiones en el mundo; Asia y el Pacífico aportan cerca del 75\% de la actual carga mundial de esta enfermedad. En el 2003, regiones en desarrollo como Bangladesh, India, Indonesia, Maldivas, Myanmar, Sri Lanka, Tailandia y Timor Leste reportaron la mayoría de casos de dengue; en junio de 2007, se notificaron brotes en las provincias de Trat, Bangkok, Chiangrai, Phetchabun, Phitsanulok, KhamkaengPhet, NakhonSawan y PhitChit, y se reportaron 58.836 casos entre enero y noviembre de 2007 (4). En la Región del Pacífico Occidental entre 2001 y 2008, los países más afectados fueron Camboya, Malasia, Filipinas y Vietnam, registran-

do 1'020.333 casos y 4.798 muertes según informes oficiales (4). En la Región
Mediterránea Oriental, cabe destacar la situación de Arabia Saudita donde se han reportado tres grandes epidemias: la primera en 1994 con 469 casos de dengue; la segunda en 2006 con 1.269 casos y la última en 2008 con 775 casos; el impacto económico y los programas de control de vectores son de gran magnitud por tener el aeropuerto más activo de la región y transportar personas provenientes de países con alta carga de la enfermedad como Indonesia, Malasia y Tailandia (4).

En la región de las américas, la subregión del Cono Sur como Argentina, Brasil, Chile, Paraguay y Uruguay entre el 2001 y 2007, reportaron el 64,6\% (2.798.601 casos), con un total de 500 muertes; siendo Brasil el país con la tasa más alta de letalidad; mientras que en la subregión de los países andinos, como Bolivia, Colombia, Ecuador, Perú y Venezuela, reportaron un total de 19\% (819.466 casos) de dengue entre 2001 a 2007. Cabe mencionar que esta es la subregión con el mayor número de casos notificados de fiebre hemorrágica por dengue: 58\% de la totalidad de los casos (61.341), con un total de 306 muertes, donde Colombia y Venezuela aportan el $81 \%$ de estos casos, sin embargo Colombia tiene la mayoría de muertes (225, equivalente a un $73 \%$ ). Para los países de América Central y México, durante estos mismos años, se reportaron 545.049 casos, con un total de 209 muertes (4).

En Colombia se han implementado estrategias educativas y programas dirigidos no solo a fortalecer los conocimientos de las personas, entre ellos los escolares sino también a mejorar las ac- 
ciones preventivas (5). En consonancia con lo anterior, Lizet Sánchez (6) argumenta que los cambios conductuales en la población se logran a largo plazo y requieren de estrategias de comunicación y movilización social de gran impacto, que promuevan y refuercen las conductas de cada uno de los miembros de la comunidad y que a su vez sean efectivas desde el punto de vista entomológico, lo cual sería un aspecto fundamental a tener en cuenta al momento de desarrollar y promover conductas preventivas en la población.

La Organización Panamericana de la Salud (OPS) ha planteado una meta para lograr la reducción de por lo menos el $30 \%$ de la tasa de letalidad por dengue (casos probables y confirmados) en el 2019 (0,05\%) (7). De igual forma, recomienda nuevos enfoques estratégicos para abordar esta problemática en los territorios, enfocándose principalmente en la promoción de la salud y la intersectorialidad para lograr acciones de control sostenibles, implementando estrategias individuales, familiares y comunitarias, empezando por los niños, a partir del rescate de conocimientos locales sobre el uso y distribución del agua, la eliminación de desechos y el saneamiento básico (8).

A nivel mundial se han aunado esfuerzos para identificar el grado de conocimiento y educación que tiene la población frente a las acciones preventivas del dengue, mediante la implementación de herramientas como las encuestas de conocimientos, actitudes y prácticas (CAP) con el objetivo de elaborar el contenido educativo de los programas de promoción para la salud y evaluación de los mismos (9).

Por todas estas razones y teniendo en cuenta la importancia de esta enfermedad, se presenta una revisión narrativa que evidencia los hallazgos sobre conocimientos, actitudes y prácticas de esta enfermedad en escolares de países en vía de desarrollo. Lo anterior, porque las medidas educativas han sido identificadas como las más eficaces en la prevención de esta enfermedad.

\section{Materiales y métodos}

Se realizó una revisión sistemática exploratoria de literatura sobre los conocimientos, actitudes y prácticas de los escolares para prevenir al dengue en países en vía de desarrollo. Las etapas de investigación fueron:

1) Búsqueda bibliográfica

2) Sistematización de datos

3) Elección de los artículos y análisis primario

4) Análisis final de los artículos

En la primera etapa se obtuvo una lista de artículos a partir de la búsqueda en: Pubmed, ScienceDirect y Scielo, en términos Thesauros: MeSH y DeCS. La búsqueda se delimito de la siguiente manera:

- Periodo: de enero de 2001 hasta diciembre de 2014.

- Idiomas: inglés y español.

- Tipo de personas analizadas en el estudio: niños escolares

- Tipo de documentos revisados: ar- 
informes de investigación, libros y tesis.

La búsqueda arrojó un listado de 70 documentos. La selección inicial se realizó con la lectura de las publicaciones que contenían los descriptores establecidos para la búsqueda, luego se dio lectura a cada artículo, excluyendo 6 artículos que estaban relacionados solo con la percepción de la población sobre el dengue y no con los conoci- mientos, actitudes y prácticas de los escolares. De los 64 artículos se excluyeron 2 que hacían referencia a la vacuna contra el dengue, 4 artículos relacionados únicamente con los factores de riesgo; 31 artículos relacionados con estudios en población y comunidades en general, es decir, sin una clasificación o énfasis específico y 11 artículos relacionados con políticas públicas. Finalmente se obtuvieron 16 artículos (Figura 1).

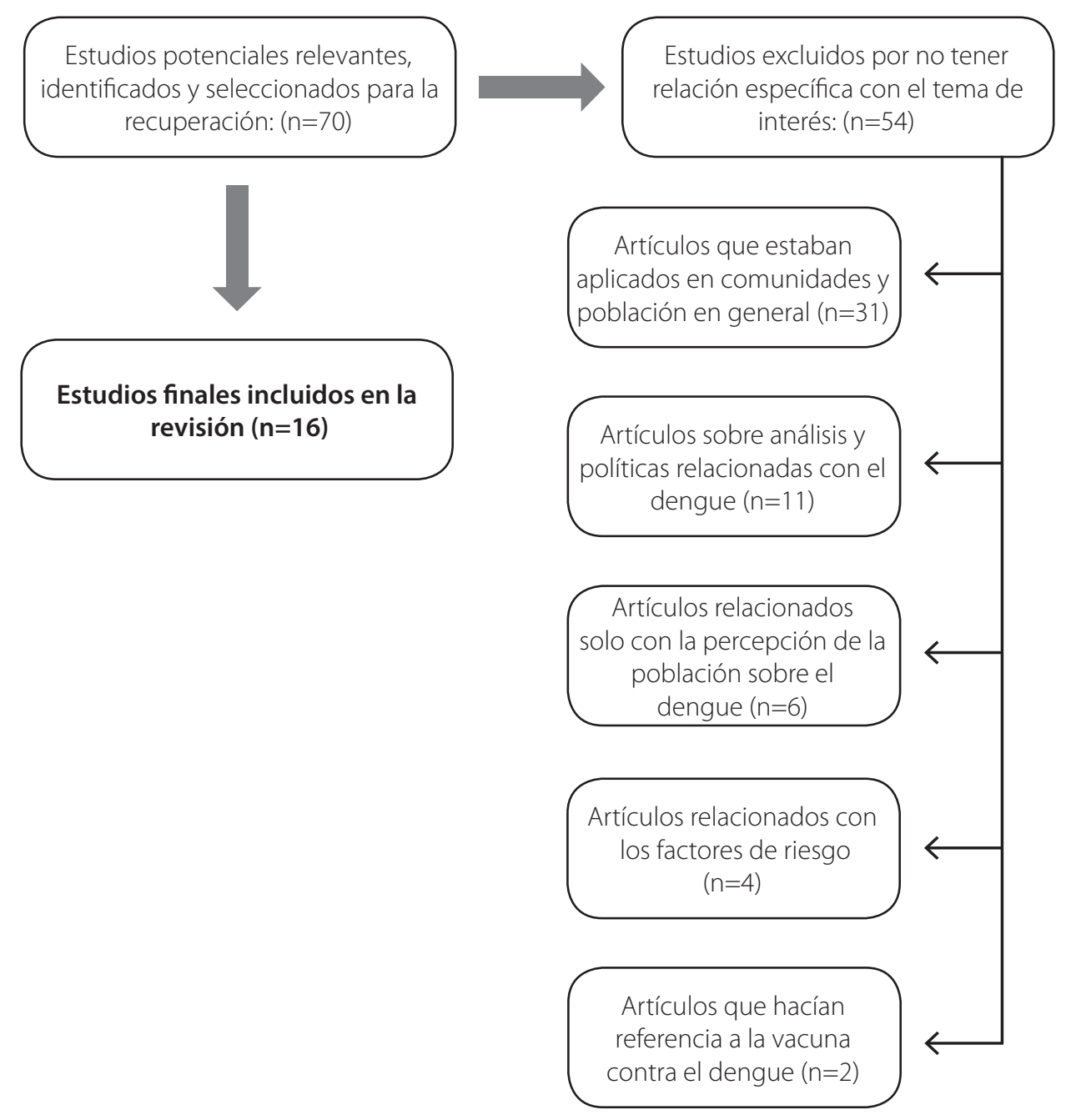

Figura 1. Proceso de selección de los estudios 
Una vez finalizada la búsqueda, se realizó la sistematización de la información, analizando la publicación de los trabajos por autor, año, diseño utilizado, instrumento, selección de la muestra, población fuente, país y análisis de la información.

\section{Resultados}

De las publicaciones identificadas (Tabla 1), el 62,5\% de los estudios (10 artículos) se realizaron entre los años 2010 y 2014. En relación con el idioma, los artículos publicados en idioma inglés $(n=8,50 \%)$ en español ( $n=8,50 \%)$. Los países que publicaron más artículos correspondieron a Tailandia y Colombia, cada uno con un total de 4 artículos (25\%). De igual forma se hizo un análisis de los países representados y número de autores por país, lo cual arrojó una totalidad de 46 autores en 10 países (Tabla 2). El país con mayor número de autores implicados en las investigaciones fue Colombia con un total de 10 autores $(21,73 \%)$ (Tabla 2). Se hizo un listado de las instituciones representadas y número de autores por institución y se obtuvo un total de 53 autores de 22 instituciones (Tabla 3).

Tabla 1. Número de estudios por año incluidos en la revisión.

\begin{tabular}{c|c|c|c|c|c|c|c|c|c|c|c}
\hline Año & 2001 & $\mathbf{2 0 0 3}$ & $\mathbf{2 0 0 4}$ & $\mathbf{2 0 0 7}$ & $\mathbf{2 0 0 9}$ & $\mathbf{2 0 1 0}$ & $\mathbf{2 0 1 1}$ & $\mathbf{2 0 1 2}$ & $\mathbf{2 0 1 3}$ & $\mathbf{2 0 1 4}$ & Total \\
\hline \# Artículos & 1 & 1 & 1 & 2 & 1 & 1 & 2 & 3 & 2 & 2 & 16 \\
\hline
\end{tabular}

Tabla 2. Países representados y número de autores

\begin{tabular}{l|c}
\hline \multicolumn{1}{c|}{ Países } & Número de autores \\
\hline Tailandia & 9 \\
Venezuela & 2 \\
México & 3 \\
Colombia & 10 \\
Sri. Lanka & 4 \\
Brasil & 5 \\
Arabia saudita & 4 \\
Honduras & 4 \\
Bangkok & 3 \\
Camboya & 2 \\
Total & $\mathbf{4 6}$ \\
\hline
\end{tabular}


INVESTIGACIONES ANDINA No. 33 Vol. 18

Tabla 3. Instituciones representadas y número de autores.

\begin{tabular}{|c|c|}
\hline Institución & Autor(es) \\
\hline University of Heidelberg, Heidelberg, Germany. & 4 \\
\hline Mahidol University, Salaya, NakhonPathom, Thailand & 3 \\
\hline RajabhatRajanagarindraUniversity, Chachoengsao, Thailand. & 1 \\
\hline NanyangTechnologicalUniversity, Singapore. & 2 \\
\hline University, Umea. & 1 \\
\hline King AbdulAziz University, Jeddah, Saudi Arabia. & 2 \\
\hline $\begin{array}{l}\text { Universidad de Carabobo, Facultad de Ciencias de la Salud, Núcleo Aragua, } \\
\text { Maracay, Venezuela. }\end{array}$ & 1 \\
\hline $\begin{array}{l}\text { Ministerio de Salud y Desarrollo Social, Dirección de Contraloría Sanitaria y } \\
\text { Saneamiento Ambiental. Maracay, Venezuela. }\end{array}$ & 1 \\
\hline Instituto Nacional de Salud Pública, Tapachula, Chiapas, México. & 3 \\
\hline $\begin{array}{l}\text { Región Metropolitana, Unidad de Control de Vectores, Departamento de } \\
\text { Salud Ambiental, Dirección General de Vigilancia de la Salud, Secretaría de } \\
\text { Salud, Tegucigalpa, Honduras. }\end{array}$ & 4 \\
\hline $\begin{array}{l}\text { Universidad Sur colombiana, Facultad de Ciencias de la Salud. Neiva, Huila, } \\
\text { Colombia. }\end{array}$ & 4 \\
\hline $\begin{array}{l}\text { Departamento de Salud Pública, Universidad Industrial de Santander. } \\
\text { Bucaramanga, Colombia. }\end{array}$ & 2 \\
\hline $\begin{array}{l}\text { Instituto Colombiano de Medicina Tropical-Universidad CES. ICMT-CES. } \\
\text { Colombia. }\end{array}$ & 2 \\
\hline Universitaria Colegio Mayor de Antioquia. Colombia. & 1 \\
\hline Departamento de Biología, Universidad Pedagógica Nacional, Colombia. & 2 \\
\hline $\begin{array}{l}\text { Universidad de cuenca. Facultad de ciencias médicas Escuela de medicina, } \\
\text { Ecuador. }\end{array}$ & 3 \\
\hline Fundación Santa Fe de Bogotá, Colombia. & 4 \\
\hline Departamento de Antropología de la Universidad de los Andes. Colombia. & 4 \\
\hline $\begin{array}{l}\text { Centro de Estudios Superiores de Caxias, Universidade Estadual do } \\
\text { Maranhão. }\end{array}$ & 4 \\
\hline Instituto Nacional de Pesquisas da Amazônia (INPA). & 1 \\
\hline National Centre for Health Promotion, Ministry of Health, Cambodia. & 1 \\
\hline $\begin{array}{l}\text { School of Psychology, Psychiatry and Psychological Medicine, Monash } \\
\text { University, Australia. }\end{array}$ & 1 \\
\hline Total de autores por institución & 53 \\
\hline
\end{tabular}

Los artículos incluidos fueron 7 $(43,75 \%)$ en el sudeste asiático, de los de Kamphaeng Sao (10), provincia de Nakhon Si Thammarat (11), y en Bangkok (12); otro estudio fue llevado a cabo en Sri. Lanka (13); uno en la ciudad de Jeddah en Arabia Saudita (9); y 
otro en Camboya (14). A nivel de Latinoamérica se incluyeron un total de 5 artículos $(31,25 \%)$ de países como Venezuela (15), Brasil (16), México (17), Honduras (18), y Ecuador (19). En Colombia se contó con 4 artículos (25\%), en las ciudades de Neiva (20), Bucaramanga (21), Medellín (22) e Ibagué
(23). Es importante tener en cuenta que la totalidad de países incluidos fueron países en vías de desarrollo. De los 16 artículos, se evidenció que el 43,75\% fueron estudios de enfoque mixto o cualitativos (7 artículos), y el 56,25\% eran estudios del paradigma empírico-analítico (9 artículos) (Tabla 4).

Tabla 4. Metodología utilizada en los estudios encontrados

\begin{tabular}{|c|c|c|c|c|c|}
\hline Autor/año & Diseño & Instrumento & Muestreo & $\begin{array}{c}\text { Población } \\
\text { fuente y país }\end{array}$ & Análisis \\
\hline $\begin{array}{l}\text { Natasha } \\
\text { Murray et. } \\
\text { al., 2014) }\end{array}$ & Descriptivo & $\begin{array}{l}\text { Cuestionario } \\
\text { Discusión } \\
\text { entre grupos } \\
\text { focales } \\
\text { Entrevista } \\
\text { semi- } \\
\text { estructurada }\end{array}$ & Aleatoria & $\begin{array}{l}\text { Estudiantes y } \\
\text { padres de un } \\
\text { colegio, de la } \\
\text { provincia de } \\
\text { Chachoengsao, } \\
\text { Tailandia. }\end{array}$ & $\begin{array}{l}\text { Estadística } \\
\text { descriptiva } \\
\text { y regresión } \\
\text { logística, análisis } \\
\text { multivariado } \\
\text { Deductivo } \\
\text { e Inductivo } \\
\text { para el análisis } \\
\text { cualitativo }\end{array}$ \\
\hline $\begin{array}{l}\text { Chanyasan- } \\
\text { ha C. et. al., } \\
\text { 2013). }\end{array}$ & $\begin{array}{l}\text { Descriptivo } \\
\text { de corte } \\
\text { transversal }\end{array}$ & Encuesta & Conveniencia & $\begin{array}{l}300 \text { estudiantes } \\
\text { de escuelas } \\
\text { secundarias en } \\
\text { Nong Kheam, } \\
\text { Bangkok, } \\
\text { Tailandia. }\end{array}$ & $\begin{array}{l}\text { Estadística } \\
\text { descriptiva }\end{array}$ \\
\hline $\begin{array}{l}\text { Suwan- } \\
\text { bamrung C. } \\
\text { Et. al.,2013) }\end{array}$ & $\begin{array}{l}\text { Corte } \\
\text { transversal }\end{array}$ & $\begin{array}{l}\text { Encuesta y } \\
\text { grupos de } \\
\text { discusión }\end{array}$ & Conveniencia & $\begin{array}{l}306 \text { estudiantes } \\
\text { de } 4^{\circ} \text { y } 6^{\circ} \\
\text { grado de } \\
5 \text { escuelas } \\
\text { primarias del } \\
\text { sub-distrito } \\
\text { Kamphaeng } \\
\text { Sao es de la } \\
\text { provincia de } \\
\text { Nakhon Si } \\
\text { Thammarat sur } \\
\text { de Tailandia }\end{array}$ & $\begin{array}{l}\text { Estadística } \\
\text { descriptiva, } \\
\text { Chi-cuadrado }\end{array}$ \\
\hline $\begin{array}{l}\text { Jayawarde- } \\
\text { ne WP. et.al., } \\
2011\end{array}$ & $\begin{array}{l}\text { Descriptivo, } \\
\text { tipo } \\
\text { intervención } \\
\text { exploratoria }\end{array}$ & Encuesta & Conveniencia & $\begin{array}{l}\text { Estudiantes de } \\
70,80^{\circ} \text { y } 9 \circ, \text { de } \\
\text { dos comunida- } \\
\text { des una urbana } \\
\text { y una rural del } \\
\text { distrito de Am- } \\
\text { para, Sri Lanka }\end{array}$ & Z-Test \\
\hline
\end{tabular}


Continuación Tabla 4. Metodología utilizada en los estudios encontrados

\begin{tabular}{|c|c|c|c|c|c|}
\hline Autor/año & Diseño & Instrumento & Muestreo & $\begin{array}{l}\text { Población } \\
\text { fuente y país }\end{array}$ & Análisis \\
\hline $\begin{array}{l}\text { Charuai- } \\
\text { Suwan- } \\
\text { bamrung, } \\
2012\end{array}$ & $\begin{array}{l}\text { Investigación } \\
\text { acción } \\
\text { participativa } \\
\text { (IAP) }\end{array}$ & $\begin{array}{l}\text { Grupos de } \\
\text { discusión y } \\
\text { cuestionarios }\end{array}$ & Propositivo & $\begin{array}{l}80 \text { niños de una } \\
\text { escuela islámica } \\
\text { de la provincia } \\
\text { de Nakhon Si } \\
\text { Thammarat sur } \\
\text { de Tailandia }\end{array}$ & $\begin{array}{l}\text { Estadística } \\
\text { descriptiva y } \\
\text { Chi -cuadrado } \\
\text { El análisis cua- } \\
\text { litativo se hace } \\
\text { con } 5 \text { catego- } \\
\text { rías de análisis } \\
\text { de percepción y } \\
\text { participación en } \\
\text { las actividades } \\
\text { lúdicas. }\end{array}$ \\
\hline $\begin{array}{l}\text { Edison Vivas, } \\
\text { Milady } \\
\text { Guevara, } \\
2003\end{array}$ & Descriptivo & $\begin{array}{l}\text { Cuestionario } \\
\text { y entrevista } \\
\text { abierta }\end{array}$ & $\begin{array}{l}\text { Aleatorio } \\
\text { sistemático }\end{array}$ & $\begin{array}{l}621 \text { escolares } \\
\text { de } 8 \text { a } 16 \text { años } \\
\text { de edad, de } \\
9 \text { escuelas } \\
\text { el municipio } \\
\text { de Girardot, } \\
\text { Estado Aragua, } \\
\text { Venezuela }\end{array}$ & $\begin{array}{l}\text { Prueba de } \\
\text { Scheffé, escala } \\
\text { de Likert }\end{array}$ \\
\hline $\begin{array}{l}\text { Paulina } \\
\text { Fajardo. } \\
\text { et.al., } 2001\end{array}$ & $\begin{array}{l}\text { Corte } \\
\text { transversal }\end{array}$ & $\begin{array}{l}\text { Entrevistas } \\
\text { estructura- } \\
\text { das, entre- } \\
\text { vistas no } \\
\text { estructuradas } \\
\text { y observa- } \\
\text { ción directa }\end{array}$ & Conveniencia & $\begin{array}{l}520 \text { hogares de } \\
\text { los escolares de } \\
\text { preescolar a } 5^{\circ} \\
\text { grado, de los } 18 \\
\text { establecimien- } \\
\text { tos Educativos } \\
\text { de la comuna } \\
\text { sur de Neiva }\end{array}$ & $\begin{array}{l}\text { Software } \\
\text { Estadístico } \\
\text { para Windows } \\
\text { y Excel. La } \\
\text { información } \\
\text { cualitativa } \\
\text { se procesó } \\
\text { manualmente } \\
\text { según } \\
\text { criterios de los } \\
\text { investigadores. }\end{array}$ \\
\hline $\begin{array}{l}\text { Celmira } \\
\text { Vesga } \\
\text { Gómez, Flor } \\
\text { Cáceres } \\
\text { Manrique. } \\
2010\end{array}$ & $\begin{array}{l}\text { Estudio de } \\
\text { intervención } \\
\text { tipo antes y } \\
\text { después }\end{array}$ & Encuesta & Conveniencia & $\begin{array}{l}\text { Estudiantes } \\
\text { líderes de } \\
\text { grupo de los } \\
\text { grados } 3^{\circ}, 4^{\circ} \\
\text { y } 5^{\circ} \text { de básica } \\
\text { primaria de } \\
\text { todos los } \\
\text { colegios } \\
\text { ubicados en } \\
\text { los dos barrios } \\
\text { de más alta } \\
\text { incidencia de } \\
\text { Dengue de } \\
\text { la Comuna } \\
\text { Norte de } \\
\text { Bucaramanga. }\end{array}$ & Prueba de $\mathrm{ji}^{2}$ \\
\hline
\end{tabular}


Continuación Tabla 4. Metodología utilizada en los estudios encontrados

\begin{tabular}{|c|c|c|c|c|c|}
\hline Autor/año & Diseño & Instrumento & Muestreo & $\begin{array}{l}\text { Población } \\
\text { fuente y país }\end{array}$ & Análisis \\
\hline $\begin{array}{l}\text { Berta } \\
\text { Restrepo .et } \\
\text { al., } 2011\end{array}$ & $\begin{array}{l}\text { Cuasi } \\
\text { experimen- } \\
\text { tal antes y } \\
\text { después }\end{array}$ & Encuesta & Conveniencia & $\begin{array}{l}66 \text { niños de } \\
\text { una institución } \\
\text { educativa } \\
\text { de Medellín. } \\
\text { Colombia }\end{array}$ & $\begin{array}{l}\text { Epilnfo, versión } \\
\text { 6,04 y Chi } \\
\text { cuadrado de } \\
\text { McNemar }\end{array}$ \\
\hline $\begin{array}{l}\text { SokrinKhun, } \\
\text { Lenore } \\
\text { Manderson. } \\
2007\end{array}$ & Etnografía & $\begin{array}{l}\text { Grupos de } \\
\text { discusión, } \\
\text { entrevistas y } \\
\text { observación }\end{array}$ & Propositivo & $\begin{array}{l}\text { Estudiantes de } \\
\text { las escuelas } \\
\text { primarias de } \\
\text { la provincia de } \\
\text { KampongCham } \\
\text { (KPC), el este de } \\
\text { Camboya }\end{array}$ & Triangulación \\
\hline $\begin{array}{l}\text { Ibrahim NK. } \\
\text { et al., } 2009\end{array}$ & $\begin{array}{l}\text { Corte } \\
\text { transversal }\end{array}$ & Encuesta & $\begin{array}{l}\text { Las } 20 \\
\text { escuelas } \\
\text { secundarias } \\
\text { femeninas } \\
\text { y las } \\
\text { estudiantes, } \\
\text { fueron } \\
\text { selecciona- } \\
\text { das con la } \\
\text { técnica de } \\
\text { Asignación } \\
\text { proporcional } \\
\text { o muestreo } \\
\text { aleatorio } \\
\text { estratificado }\end{array}$ & $\begin{array}{l}\text { Estudiantes, } \\
\text { profesores, } \\
\text { supervisores. } \\
\text { De } 20 \text { escuelas } \\
\text { femeninas de } \\
\text { los grados } 1^{\circ}, \\
2^{\circ} \text { y } 3^{\circ} \text { año) de } \\
\text { Jedahh, Arabia } \\
\text { Saudita }\end{array}$ & $\begin{array}{l}\text { Multivariado } \\
\text { estratificado } \\
\text { SPSS software } \\
\text { program } \\
\text { (version13.0; } \\
\text { SPSS Inc, } \\
\text { Chicago, III., } \\
\text { USA) }\end{array}$ \\
\hline $\begin{array}{l}\text { José Luis } \\
\text { Torres. et.al., } \\
2014\end{array}$ & $\begin{array}{l}\text { Corte } \\
\text { transversal }\end{array}$ & Encuesta & Aleatorio & $\begin{array}{l}1.562 \text { alumnos } \\
\text { de } 5^{\circ} \text { y } 6^{\circ} \text { grado } \\
\text { de escuelas } \\
\text { primarias. } \\
\text { Tapachula, } \\
\text { Chiapas, } \\
\text { México }\end{array}$ & $\begin{array}{l}\text { Program } \\
\text { Stat - View SAS, } \\
\text { Institute, } \\
\text { Escalatipolikert }\end{array}$ \\
\hline $\begin{array}{l}\text { Gustavo } \\
\text { Adolfo Ávila } \\
\text { Montes. } \\
2014\end{array}$ & $\begin{array}{l}\text { Ensayo de } \\
\text { campo } \\
\text { comunitario }\end{array}$ & $\begin{array}{l}\text { Encuesta, } \\
\text { Entrevista y } \\
\text { Observación } \\
\text { participativa }\end{array}$ & Conveniencia & $\begin{array}{l}\text { Estudiantes, } \\
\text { profesores, } \\
\text { madres } \\
\text { de cuatro } \\
\text { comunidades } \\
\text { urbanas } \\
\text { marginales } \\
\text { que poseían } \\
\text { escuelas } \\
\text { primarias } \\
\text { públicas, en } \\
\text { Honduras. }\end{array}$ & $\begin{array}{l}\text { Epi-Info 6.04ab. } \\
\text { El análisis } \\
\text { estratificado y } \\
\text { multifactorial } \\
\text { paquete SPSS. Y } \\
\text { para el análisis } \\
\text { cualitativo } \\
\text { por medio de } \\
\text { Matrices de ejes } \\
\text { temáticos. }\end{array}$ \\
\hline
\end{tabular}


INVESTIGACIONES ANDINA No. 33 Vol. 18

Continuación Tabla 4. Metodología utilizada en los estudios encontrados

\begin{tabular}{|c|c|c|c|c|c|}
\hline Autor/año & Diseño & Instrumento & Muestreo & $\begin{array}{l}\text { Población } \\
\text { fuente y país }\end{array}$ & Análisis \\
\hline $\begin{array}{l}\text { Lucas } \\
\text { Andrés } \\
\text { Alcalá } \\
\text { Espinosa, } \\
\text { Hernández } \\
\text { Pichardo } \\
\text { Análida. } \\
2012\end{array}$ & $\begin{array}{l}\text { Enfoque } \\
\text { mixto }\end{array}$ & $\begin{array}{l}\text { Observacio- } \\
\text { nes participa- } \\
\text { tivas activas, } \\
\text { revisión } \\
\text { documental, } \\
\text { encuesta } \\
\text { CAP y una } \\
\text { entrevista } \\
\text { semi estruc- } \\
\text { turada, escala } \\
\text { tipo Likert. }\end{array}$ & Conveniencia & $\begin{array}{l}\text { Estudiantes } \\
\text { de } 6{ }^{\circ} \text { grado, y } \\
\text { familiares. } \\
\text { Colegio en } \\
\text { Ibagué, } \\
\text { Colombia }\end{array}$ & $\begin{array}{l}\text { Escala tipo } \\
\text { likert. } \\
\text { Triangulación } \\
\text { de la } \\
\text { información } \\
\text { obtenida en la } \\
\text { encuesta CAP }\end{array}$ \\
\hline $\begin{array}{l}\text { María } \\
\text { Eugenia } \\
\text { Lozano. et. } \\
\text { al. } 2012 \text {. }\end{array}$ & Descriptivo & Encuesta & $\begin{array}{l}\text { Probabilístico } \\
\text { aleatorio }\end{array}$ & $\begin{array}{l}640 \text { estudiantes } \\
\text { de tercer año } \\
\text { de bachillerato } \\
\text { en los colegios } \\
\text { fiscales } \\
\text { Machala, } \\
\text { Ecuador. }\end{array}$ & Software SPSS \\
\hline $\begin{array}{l}\text { Juliana } \\
\text { Trindade } \\
\text { Bezerra. et. } \\
\text { al. } 2011\end{array}$ & Descriptivo & Cuestionario & Conveniencia & $\begin{array}{l}700 \text { Estudiantes } \\
\text { de } 14 \text { escuelas } \\
\text { públicas de } \\
7 \text { barrios de } \\
\text { la ciudad de } \\
\text { Caxias, estado } \\
\text { de Maranhao. }\end{array}$ & $\begin{array}{l}\text { Chi-cuadrado - } \\
\text { software Systat, } \\
\text { version } 10.2\end{array}$ \\
\hline
\end{tabular}

Por otra parte en el 50\% de los estudios analizados (8 artículos), midieron los índices larvarios e hicieron referencia a la correlación de estos con la presencia de casos de dengue. La totalidad de artículos en donde se emplearon estrategias educativas de intervención (9 artículos) equivalentes a un 56,25\%, evidenciando un aumento en los conocimientos de los estudiantes luego de padecer la enfermedad. Así como se evidenció que solo un estudio $(6,25 \%)$ comparaba los conocimientos, actitudes y prácticas de un grupo de estudiantes, en dos barios con diferentes condiciones sociales y urbanas.

\section{Recopilación bibliográfica (Tabla 4)}

Los estudios realizados en el subdistrito Kamphaeng Sao (10), Tailandia; el otro en la provincia de Nakhon Si Thammarat sur de Tailandia (11), en Bangkok (12), Sri. Lanka (13), y Jeddah (9), concluyeron que los programas de salud deben ser permanentes, continuos y de una gran intensidad con énfasis en la prevención y en las prácticas de control orientadas a la disminución de la enfermedad y a mejorar las buenas prácticas en salud para la prevención de la misma. 
Sin embargo, se evidenció que en estudios como el de Camboya (14), los escolares no poseían suficientes conocimientos, actitudes y prácticas relacionadas con la efectividad de la interrupción colectiva del ciclo de vida de los mosquitos transmisores de dengue, incluso después de haber implementado la estrategia dada por el Ministerio de Educación y el Ministerio de Salud de este país.

Uno de los estudios realizado en la provincia de Chachoengsao, Tailandia (3) enfocado a modificar las prácticas de la población escolar, evidenció que las limitaciones económicas de los padres son un factor decisivo en la prevención del dengue, ya que los escolares estudiados, no pudieron continuar con la práctica del uso de uniformes impregnados de insecticida (90\% de los estudiantes la iniciaron) porque al cabo de un tiempo el 59.9\% de estos padres manifestaron que no lo seguirían usando, debido a que no contaban con los recursos económicos para seguir comprando los uniformes (3).

Por otra parte, la investigación llevada a cabo en Bangkok (12) además de conocimientos, evaluó la percepción y se pudo observar que los estudiantes mostraron un buen nivel de conocimiento y porcentajes más bajos en cuanto a percepción del dengue y comportamientos preventivos. Un estudio realizado en la ciudad de Jeddah (9) diferenció los conocimientos entre estudiantes, profesores y supervisores y se encontró que los estudiantes obtuvieron la más baja puntuación en comparación con los otros dos grupos. Aquellos estudiantes mayores de 17 años, hijos de madres alfabetizadas y que registraron al menos un caso de dengue en su familia obtuvieron mayor puntaje en conocimientos (9).

Los estudios hallados a nivel de Latinoamérica, concluyeron que se debe hacer un mayor énfasis en las campañas educativas, puesto que son necesarias para reforzar la conducta y cambiar las prácticas de la población frente a la prevención del dengue. Lo anterior es probable dado que existe evidencia de que las campañas de prevención realizadas a través de medios de comunicación, especialmente la televisión son efectivas (16).

Es importante revelar que solo un estudio en Latinoamérica, llevado a cabo en el municipio de Caxias, estado de Maranhão en Brasil (16), hizo una correlación entre dos barrios de distintas condiciones sociales, donde se encontró que los escolares pertenecientes al barrio con mejores condiciones sociales y urbanas tenían conceptos más claros sobre el dengue y su prevención.

Los estudios encontrados en Colombia, específicamente en Neiva (20), Bucaramanga (21), Medellín (22) e Ibagué (23), demostraron que la implementación de estrategias educativas debe ser perdurable en aquellas instituciones en las ciudades donde la enfermedad es endémica. También se deben reforzar conocimientos del ciclo de vida del vector, su papel en la transmisión y el rol de la comunidad como interventor activo del ambiente en el cual se desarrolla (23). 
En cuanto al estudio que se llevó a cabo en la ciudad de Bucaramanga (21), fue el único, donde los escolares se comprometieron por escrito a implementar actividades encaminadas a la prevención del dengue con familiares y vecinos. Además, se hizo un seguimiento por cuatro meses $y$ se realizó una visita domiciliaria para ver si los niños habían cumplido con su compromiso. Al finalizar el estudio, ellos realizaron sus compromisos e hicieron más actividades con creatividad e ingenio (21).

La investigación que se realizó en la ciudad de Neiva (20), Colombia, mostró la presencia de un patrón cultural en la forma en la que ellos se refieren a la enfermedad y a la manera en la que la abordan. Por tanto, la percepción de la comunidad debe orientar también la formulación y el desarrollo de los programas educativos (22).

Para enfatizar, los estudios llevados a cabo en una escuela islámica del sur de Tailandia (10,11), en Honduras (18), Bucaramanga (21), Colombia, México (17), Medellín (22), y Sri Lanka (13), evidenciaron que los conocimientos mínimos que mostraron los estudiantes, estaban directamente relacionados con los altos índices larvarios encontrados en sus hogares y en las escuelas. Sin embargo, posterior a la implementación de la estrategia educativa se mostró un incremento significativo en cuanto al conocimiento sobre aspectos básicos y claves del dengue, el reconocimiento del vector y sus sitios de reproducción, así como de los síntomas y las prácticas de prevención. De igual forma, se evidenció una reducción de fuentes de criaderos de los mosquitos, lo que conllevó a una disminución de los índices larvarios, lo que redujo también la cantidad de casos de dengue.

\section{Discusión}

\section{Conocimientos}

En cuanto a conocimientos, las dos investigaciones llevadas a cabo en el sur de Tailandia $(11,12)$ demostraron que el incremento del riesgo de contraer dengue, está directamente relacionado con los conocimientos mínimos de los estudiantes, junto con el alto índice larvario encontrado en sus hogares y en las escuelas (8). Además, en otros países como Venezuela (15), México (17) y en Medellín-Colombia (22), mostraron que las estrategias educativas que usaron herramientas participativas, lúdicas y divertidas suelen ser muy eficaces para prevenir y controlar este tipo de enfermedades (22). Sin embargo, el estudio que se llevó a cabo en Ecuador (19) reveló un buen nivel de conocimientos en escolares.

\section{Actitudes}

En cuanto a las actitudes, el estudio llevado a cabo en Ecuador (19) evidenció que la actitud y los conocimientos de los escolares frente al dengue están interrelacionados cuando son positivos; sin embargo, el estudio realizado en la ciudad de Ibagué (23), Tolima, a pesar que mostró también una actitud positiva frente a la prevención de la enfermedad, el resultado sobre los conocimientos y prácticas fue contrario (23). 


\section{Prácticas}

De igual forma, en Tailandia (11) y Honduras (19) en cuanto a prácticas, los hogares de los escolares realizaron un manejo seguro del agua, así como un aumento en la gestión para la adecuada recolección de basuras, lo que generó una disminución de los índices larvarios (11); mientras que el estudio realizado en el distrito de Ampara en Sri. Lanka (13) demostró que, un año después de ejecutar una intervención educativa en escolares y otros grupos humanos, la cantidad de casos de dengue en el área urbana y rural pueden ser disminuidos hasta en un $73 \%$ (13).

En cuanto a los alcances de esta revisión, se logró evidenciar que las estrategias educativas y programas en salud, dirigidos a fortalecer los conocimientos y prácticas de los escolares frente al dengue, ayudan a transformar sus actitudes y comportamientos y por ende a mejorar las acciones preventivas frente a esta enfermedad. También permitió evidenciar que la aplicación de los cuestionarios sobre conocimientos, actitudes y prácticas sí son una herramienta útil para abordar los resultados de la implementación de programas educativos.

Las limitaciones del estudio se centraron en la selección de los artículos ya que existe una cantidad considerable de estudios sobre conocimientos, actitudes y prácticas frente al dengue aplicados a poblaciones en general más no exclusivamente a escolares.

\section{Conclusiones}

Los cambios individuales y colectivos a nivel comportamental parten de programas eficaces de concientización y enseñanza de la responsabilidad individual pero también de la corresponsabilidad través de la participación social. En el caso de los escolares, es importante que los conocimientos y prácticas de prevención y control del dengue se adquieran a través de juegos y actividades lúdicas que propendan la adquisición de hábitos saludables.

Los estudios de conocimientos, actitudes y prácticas en escolares han sido publicados, en su mayoría, entre los años 2010 y 2014 siendo Tailandia y Colombia los países en vía de desarrollo con mayor número de publicaciones. Asimismo, la mitad de los estudios correlacionan los índices larvarios con la presencia de la enfermedad. Se demuestra que el proceso educativo es más eficiente en la edad temprana, lo que hace pensar que las estrategias de intervención y programas de prevención deben aplicarse en la educación formal preescolar y escolar, ya que los niños pueden ser grandes divulgadores y promotores de conocimientos y prácticas desde sus hogares, colegios, familias y comunidades, puesto que pueden promover hábitos en las generaciones anteriores que ayuden con la prevención del dengue, y lograr que la población en general asuma realmente un rol como agente de cambio (22).

Respecto a los conocimientos de los escolares para prevenir el dengue se evidencio que eran suficientes, pero las ac- 
titudes y prácticas insuficientes dada la falta de continuidad de los programas y la ausencia de prácticas en la familia. De igual forma, se pudo evidenciar que la implementación de estrategias educativas que aumentan los conocimientos, actitudes y prácticas de los niños para prevenir la enfermedad, se enfatizan en el auto-cuidado desde escuelas y hogares. Con lo anterior, se puede concluir que estos nuevos conocimientos y habilidades adquiridas por ellos, podrían ser aplicados en sus respectivas comunidades con el fin de promover la prevención de la enfermedad como una estrategia de empoderamiento (24).

\section{Referencias}

1. Instituto Nacional de Salud. Informe Epidemiológico Nacional 2012, enfermedades transmisibles, enfermedades transmitidas por vectores, Chagas, Dengue, Fiebre Amarilla, Leishmaniasis, Malaria. [Internet]. [Consultado el 25 abril de 2015]. Disponible en:http://www.ins. gov.co/lineas-de-accion/Subdireccion-Vigilancia/Informe $\% 20$ de $\% 20$ Evento $\% 20$ Epidemiolgico/Informe\%20final\%202012/ Final\%202012\%20Enf.\%20Transmisibles\%20ETV.pdf.

2. Gubler, D. J. Dengue/Dengue Haemorrhagic Fever: History and Current Status, in New Treatment Strategies for Dengue and Other Flaviviral Diseases: Novartis Foundation Symposium 277. [Internet]. USA: Novartis Foundation; 2006 [Consultado el 10 de abril de 2015]. Disponible en: http://onlinelibrary.wiley.com/ book/10.1002/0470058005

3. Murray, N., Jansarikij, S., Olanratmanee, P., Maskhao, P., Souares, A., Wilder-Smith, A., Kittayapong, P., Louis, V. Acceptability of impregnated school uniforms for dengue control in Thailand: a mixed methods approach. Glob Health Action 2014. 7(0); 24887
4. World Health Organization. Dengue, guías para el diagnóstico, tratamiento, prevención y control. [Internet]. [Consultado el 10 de abril de 2015]. Disponible en: http://www.bvsde.paho.org/cursoa_dengue/e/pdf/dengue_oms_2009.pdf.

5. Castro Rodríguez R, Carrasquilla G, Porras A, Galera-Gelvez K, Lopez Yescas JG, Rueda-Gallardo JA. The Burden of Dengue and the Financial Cost to Colombia, 2010-2012.Am J Trop Med Hyg. 2016; pii: 15-0280

6. Sánchez L, Pérez D, Alfonso L, Castro $M$, Sánchez LM, Van der Stuyft $P$, et al. Estrategia de educación popular para promover la participación comunitaria en la prevención del dengue en Cuba. Rev Panam Salud Pública. 2008; 24(1):61-9.

7. Pan American Health Organization. En pro de la salud: Desarrollo sostenible y equidad: Plan Estratégico de la OPS 2014-2019. [Internet]. [Consultado el 15 de abril de 2015].Disponible en: http:// www. paho.org/hq/index. php?option=com_docman\&task=doc_download\&gi-

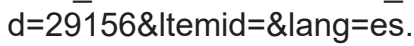


8. Instituto Nacional de salud. Gestión para la vigilancia entomológica y control de la transmisión de dengue. [Internet]. [Consultado el 20 de junio de 2015]. Disponible en: http://www.ins.gov.co/temas-de-interes/Dengue/03\%20Vigilancia\%20entomo\%20dengue.pdf

9. Ibrahim NK, Al-Bar A, Kordey M, AlFakeeh A. Knowledge, attitudes, and practices relating to Dengue fever among females in Jeddah highschools. J Infect Public Health. 2009;2(1):30-40

10. Suwanbamrung Ch, Promsupa S, Doungsin T, Tongjan S. Risk factors related to dengue infections in primary school students: exploring students' basic knowledge of dengue and examining the larval indices in southern Thailand. J Infect Public Health. 2013;6(5):347-57

11. Suwanbamrung Ch. Children's basic knowledge and activities for dengue problem solution: an islamic religious school, Southern Thailand. Asian Pac J Trop Disease. 2012;8(3): $456-64$

12. Chanyasanha C, Han MM, Teetipsatit $S$. Dengue hemorrhagic fever knowledge, perception, and preventive behavior among secondary school students in Bangkok.". J Med Assoc Thai.2013; 96 (5):S14-24.

13. Jayawardene WP, Lohrmann DK, Youssef Agha AH, Nilwala DC. Prevention of dengue Fever: an exploratory school-community intervention involving students empowered as change agents 2011; 81(9)566-73.

14. Khun S, Manderson L. Community and School-Based Health Education for Dengue Control in Rural Cambodia: A Process Evaluation. PLoS Negl Trop Dis. 2007; 1(3) e143.

15. Vivas E, Guevara de Sequeda M. Un juego como estrategia educativa para el control de Aedes aegypti en escolares venezolanos. Pan Am J Public Health.2003; 14(6): 394-401.
16. TrindadeJ, Soares-da-Silva J, Silva S, Wanderli P, Soares V. Evaluation of student's knowledge as a contribution to dengue control programs. Ciência \& Saúde Coletiva 2011; 16(11):4367-73.

17. Torres JL, Ordóñez JG, Vázquez-Martínez MG. Conocimientos, actitudes y prácticas sobre el dengue en las escuelas primarias de Tapachula, Chiapas, México. Rev Panam Salud Pública. 2014; 35(3):214-8.

18. Ávila Montes GA, Martínez M, Sherman C, Fernández Cerna E. "Evaluación de un módulo escolar sobre dengue y Aedes aegypti dirigido a escolares en Honduras.Pan Am J PublicHealth. 2004; 16(2):84-94.

19. Lozano M, Martínez B, Siavichay P. Conocimientos, actitudes y prácticas sobre dengue en los estudiantes de tercer año de bachillerato en los colegios fiscales: 9 de octubre, Atahualpa, Amazonas, Mario Minuche Murillo, Kleber Kranco Cruz, Juan Montalvo, República de Perú, Red educativa Rotary Club, El Oro, República del Ecuador, de Machala, 2012. [Trabajo de pregrado Medicina]. Cuenca, Ecuador: Universidad de Cuenca. Facultad de Medicina. 2013.

20. Fajardo P, Monje CA, Lozano G, Realpe O y Hernández LE. Nociones populares sobre "dengue" y "rompehuesos", dos modelos de la enfermedad en Colombia. Pan Am J Public Health 2001; 10(3): 161-9.

21. Cáceres F, Vesga C. Eficacia de la educación lúdica en la prevención del Dengue en escolares. Rev. Salud pública. 2010; 12(4): 558-69.

22. Restrepo BN, Pineda JM, Parra-Henao GJ. Aplicación y evaluación de materiales educativos para la prevención del dengue en una institución educativa de Medellín, Colombia. Rev. CES Med. 2011; 25(1): 31-41.

23. Alcalá Espinosa LA, Hernández Pichardo A. Conocimientos, actitudes y prácticas frente a la prevención del dengue, en estudiantes del grado 6-11 de una 
INVESTIGACIONES ANDINA No. 33 Vol. 18

institución educativa en Ibagué-Tolima 2012. Biografía: Escritos sobre la Biología y su Enseñanza. 2012; 5(8): 9-31.
24. Sanabria G. Participación social y comunitaria: Reflexiones. Rev. Cubana salud pública; 2001; 27: 89-95 